\title{
Tunneling in epitaxial Fe/Si/Fe structures with strong antiferromagnetic interlayer coupling
}

\author{
R. R. Gareev, ${ }^{\text {a) }}$ L. L. Pohlmann, S. Stein, D. E. Bürgler, ${ }^{\text {b) }}$ and P. A. Grünberg \\ Institut für Festkörperforschung, Foschungszentrum Jülich GmbH, D-52425 Jülich, Germany \\ M. Siegel \\ Institut für Schichten und Grenzflächen, Foschungszentrum Jülich GmbH, D-52425 Jülich, Germany
}

(Presented on 14 November 2002)

\begin{abstract}
$\mathrm{Fe}(5 \mathrm{~nm}) / \mathrm{Si}(0.8-2 \mathrm{~nm}) / \mathrm{Fe}(5 \mathrm{~nm})$ structures are grown by molecular-beam epitaxy on $\operatorname{Ag}(001)$ buffered GaAs substrates. Ferromagnetic tunneling junctions with crossed electrodes and junction areas ranging from 22 to $225 \mu \mathrm{m}^{2}$ are patterned using photolithography. Antiparallel alignment of the magnetizations due to antiferromagnetic interlayer coupling, which is confirmed by longitudinal magneto-optical Kerr effect hysteresis loops, exists for the whole range of spacer thicknesses. Transport properties in current perpendicular to the sample plane geometry are examined by the four-point method in the temperature range from $4 \mathrm{~K}$ to room temperature. As a function of spacer thickness, the junctions show a strong increase of the resistance times area product from $\approx 1 \Omega \mu \mathrm{m}^{2}$ to more than $10 \mathrm{k} \Omega \mu \mathrm{m}^{2}$. The $d I / d V-V$ curves are parabolic and asymmetric and thus characteristic for trapezoidal tunneling barriers. The mean barrier heights derived from Brinkman fits range from 0.3 to $0.8 \mathrm{eV}$. The zero-bias resistance of the tunneling junctions moderately decreases with temperature by less than $10 \%$ over the whole measured temperature range. All these transport properties fulfill the necessary and sufficient criteria for elastic tunneling. () 2003 American Institute of Physics. [DOI: 10.1063/1.1543989]
\end{abstract}

\section{INTRODUCTION}

Epitaxial $\mathrm{Fe} / \mathrm{Si} / \mathrm{Fe}$ structures are interesting due to strong antiferromagnetic $(\mathrm{AF})$ interlayer coupling and its interplay with transport properties, both of which are sensitive to preparation conditions. ${ }^{1-4}$ Recently, we have found that insulating-type, highly resistive spacers can be prepared by a certain deposition procedure. ${ }^{5-8}$ Corresponding $\mathrm{Fe} / \mathrm{Si} / \mathrm{Fe}$ structures reveal very strong AF interlayer coupling with a total coupling strength in excess of $5 \mathrm{~mJ} / \mathrm{m}^{2},{ }^{5}$ which could be further increased to $8 \mathrm{~mJ} / \mathrm{m}^{2}$ by inserting thin epitaxial and metallic $\mathrm{FeSi}^{6}$ boundary layers at interfaces. ${ }^{7}$ For combined semiconducting/metallic epitaxial spacers (i.e., nominally pure $\mathrm{Si} / \mathrm{Fe}_{0.5} \mathrm{Si}_{0.5}$ ), the main impact to $\mathrm{AF}$ coupling arises from the semiconducting part of the spacer. ${ }^{8}$ Hence, we came to the conclusion that the strong and exponentially decaying AF coupling arises from Si-rich spacers, in qualitative agreement with the quantum interference model of exchange coupling across insulating spacers. ${ }^{9}$

The behavior of interlayer coupling indicates that nominally pure $\mathrm{Si}$ spacers are effectively not homogeneous. For a nominal spacer thickness $t$ less than several monolayers, the interlayer coupling becomes ferromagnetic (FM) very likely due to conducting pinholes formed by interdiffusion at the interfaces. ${ }^{5,7}$ For thicker spacers, the effect of pinholes andaccordingly - FM coupling are suppressed.

The resistivity in current perpendicular to the sample plane (CPP) geometry is expected to yield additional and clear information whether a Si-rich spacer is metallic or insulating. Hence, the question is whether the transport in

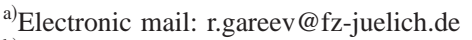

b) Author to whom correspondence should be addressed; electronic mail: d.buergler@fz-juelich.de
}

highly resistive spacers is due to elastic tunneling, or whether it arises from an additional channel of conductivity across submicron-sized pinholes, as was pointed out in Refs. 10 and 11. For this reason we examine for our structures the validity of the necessary and sufficient criteria for direct elastic tunneling, ${ }^{11}$ i.e., (i) strong and exponential increase of the resistance with $t$, (ii) parabolic dependence of conductivity versus bias voltage, and-most decisive-(iii) small and negative temperature coefficient of the zero-bias resistance.

\section{EXPERIMENTAL PROCEDURES}

The preparation procedure of $\mathrm{Fe}(5 \mathrm{~nm}) / \mathrm{Si}(0.8-2 \mathrm{~nm}) /$ $\mathrm{Fe}(5 \mathrm{~nm})$ wedge-type epitaxial samples on $\operatorname{Ag}(150 \mathrm{~nm}) / \mathrm{Fe}(1$ $\mathrm{nm}) / \mathrm{GaAs}(100)$ buffer systems has been described elsewhere. ${ }^{5,6}$ We measure longitudinal magneto-optical Kerr effect (MOKE) hysteresis loops to verify the presence of $\mathrm{AF}$ coupling, which is reflected by zero remanent magnetization. The external magnetic field is applied along an easy axis of the Fe layers. Additionally, we prepared structures with constant spacer thickness and checked the antiparallel alignment by superconducting quantum interference device magnetom-

TABLE I. Junction area $A$, nominal spacer thickness $t$, junction resistance $R$, and the parameters derived from Brinkman fits: mean barrier height $\phi$, barrier asymmetry $\Delta \phi$, and the fitted barrier thickness $t_{\mathrm{eff}}$ for various junctions labeled $J 1$ to $J 6$.

\begin{tabular}{ccccccc}
\hline \hline Junction & $A\left(\mu \mathrm{m}^{2}\right)$ & $t(\mathrm{~nm})$ & $R(\Omega)$ & $\phi(\mathrm{eV})$ & $\Delta \phi(\mathrm{eV})$ & $t_{\text {eff }}(\mathrm{nm})$ \\
\hline$J 1$ & 22 & 1.54 & 307 & 0.35 & -0.35 & 1.83 \\
$J 2$ & 22 & 1.70 & 23.4 & 0.54 & -0.36 & 1.22 \\
$J 3$ & 100 & 1.48 & 26.0 & 0.33 & 0.05 & 1.74 \\
$J 4$ & 100 & 1.54 & 5.6 & 0.43 & -0.18 & 1.35 \\
$J 5$ & 100 & 1.64 & 223 & 0.78 & -0.30 & 1.44 \\
$J 6$ & 225 & 1.70 & 5.3 & 0.36 & -0.10 & 1.55 \\
\hline \hline
\end{tabular}



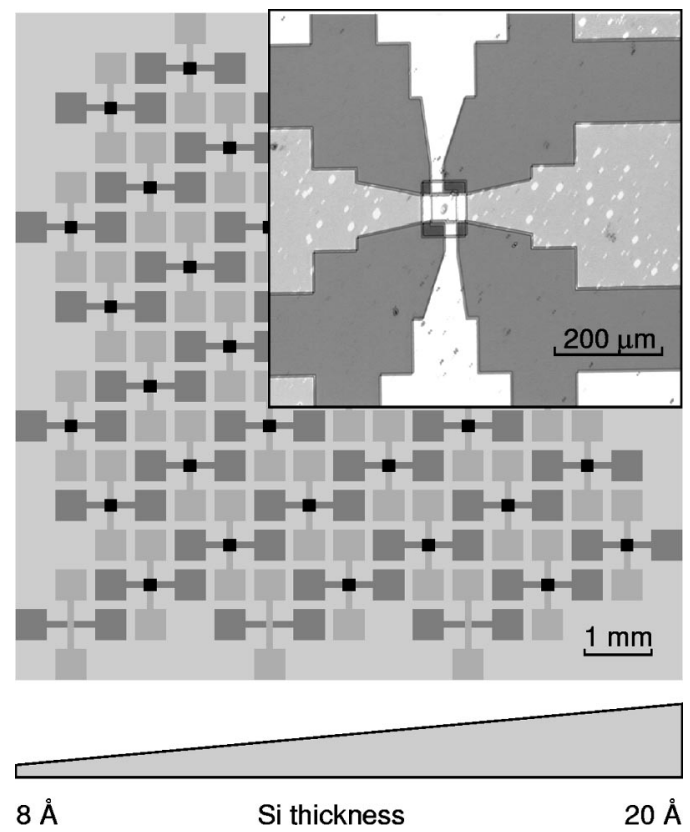

FIG. 1. Layout of the sample with a wedge-type Si spacer layer resulting in junctions with different spacer thicknesses. The inset shows a photograph of a typical patterned junction.

etry, where we can apply higher magnetic fields and saturate the samples. After these magnetic characterizations, 10 $\times 10 \mathrm{~mm}^{2}$-sized, wedge-type samples are patterned using photolithography, ion-beam etching, and lift-off technique to obtain CPP junctions with different spacer thicknesses, which are all deposited under the same growth conditions. We use crossed contacts, where the upper electrode is formed by depositing a 300-nm-thick $\mathrm{Cu}$ layer. The patterned 150 $\mathrm{nm}$-thick silver buffer layer serves as a bottom electrode. The sheet resistances $R$ of both electrodes are about $0.1 \Omega$ and thus significantly smaller than the resistance of the tunneling junctions in CPP geometry (Table I), such that current distribution effects are diminished. ${ }^{12}$ Insulation of the electrodes is achieved by deposition of a 250 -nm-thick Si-oxide layer. Finally, we define junctions of rectangular shape ranging in area $A$ from 22 to more than $200 \mu \mathrm{m}^{2}$. The layout of the patterned sample is shown in Fig. 1. A typical patterned junction is shown in the inset. After patterning, voltage and cur-

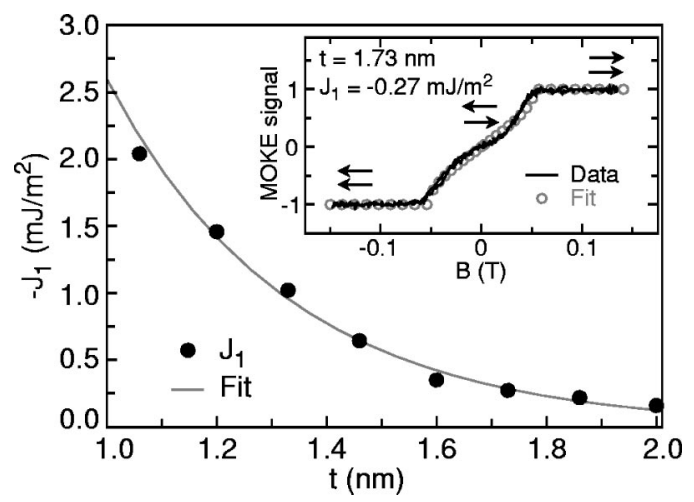

FIG. 2. AF coupling constant $J_{1}$ vs spacer thickness $t$. The fitted curve yields a decay length of $0.33 \mathrm{~nm}$. Inset: Experimental and fitted longitudinal MOKE hysteresis curves clearly show antiparallel alignment due to $\mathrm{AF}$ coupling and yield $J_{1}=-0.27 \mathrm{~mJ} / \mathrm{m}^{2}$.

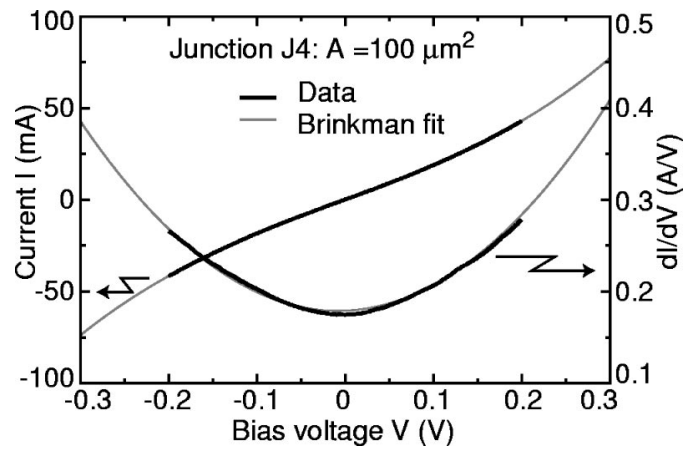

FIG. 3. Measured (black) and fitted (gray) $I-V$ and $d I / d V$ curves of junction $J 4$.

rent leads suitable for four-point transport measurements are connected by ultrasonic bonding. We measure the $I-V$ characteristics of junctions with different areas $A$ and nominal spacer thicknesses $t$ (see Table I).

\section{RESULTS AND DISCUSSION}

The thickness dependence of the AF interlayer coupling strength $J_{1}$ of our structures is shown in Fig. 2. $\left|J_{1}\right|$ decays exponentially with $t$ with a decay length of about $0.3 \mathrm{~nm}$. Finally, for $t \approx 2 \mathrm{~nm}$ the AF coupling strength decreases to $\left|J_{1}\right| \approx 0.1 \mathrm{~mJ} / \mathrm{m}^{2}$. The zero-field antiparallel alignment is observed in the whole range of temperatures and for all spacer thicknesses.

A representative $I-V$ curve taken at room temperature and the corresponding $d I / d V-V$ curve are presented in Fig. 3 and show the typical tunneling-type behavior. The $d I / d V$ $-V$ curve is parabolic with its minimum away from $V=0$. These features are characteristic for tunnel junctions with asymmetric barriers and indicate different conditions at the diffused $\mathrm{Fe} / \mathrm{Si}$ and $\mathrm{Si} / \mathrm{Fe}$ interfaces, probably due to different interfacial densities of states and/or transmission probabilities of the carriers. There is no evidence for a conductivity anomaly near $V=0$, as previously reported for ferromagnetic junctions with Al-oxide spacers and related to inelastic scattering assisted by magnons and impurities. ${ }^{13}$

Similar $I-V$ curves can occur when transport is due to another conductivity channel, namely submicron-sized pinholes, which can mimic elastic tunneling. ${ }^{10}$ As we will show below based on an analysis of the temperature dependence of the resistance, this metallic-type channel gives no significant contribution.

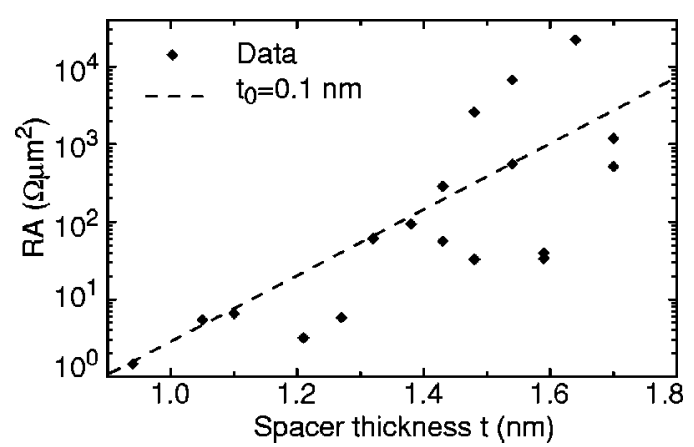

FIG. 4. Dependence of the resistance times area product $R A$ on the nominal spacer thickness $t$ obtained from a wedge-type $\mathrm{Fe} / \mathrm{Si} / \mathrm{Fe}$ structure. The dashed line corresponds to a decay length $t_{0}=1 \AA$. 


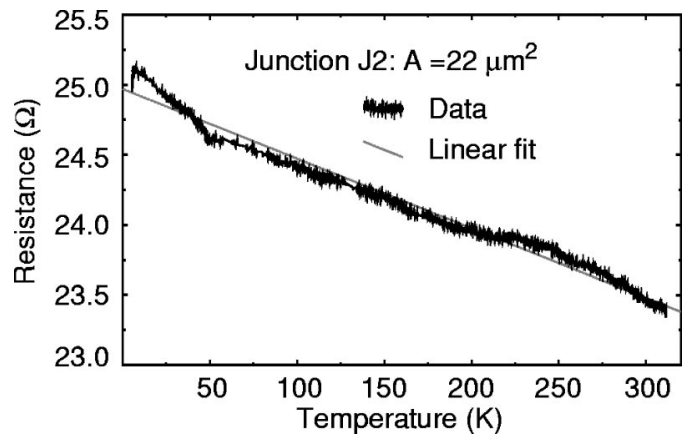

FIG. 5. Dependence of the resistance on temperature for junction $J 2$. The solid line is a linear fit yielding a temperature coefficient of -5 $\times 10^{-3} \Omega \mathrm{K}^{-1}$.

In Fig. 4 we show the resistance times area product $R A$ vs $t$ on a semilogarithmic scale. The value of $R A$ increases at room temperature strongly with $t$ by more than four orders of magnitude, while $t$ only approximately doubles. The decay length $t_{0} \approx 1 \AA$ (dashed line in Fig. 4) is significantly shorter compared to corresponding decay lengths for structures with amorphous Si spacers. ${ }^{14}$ Note, that the AF exchange coupling across epitaxial, $\mathrm{Si}$ - rich spacers is also a short-range interaction and decays with a very similar decay length as the tunneling. 5

At present, we observe tunneling-type $I-V$ curves only for $t>1.5 \mathrm{~nm}$, where the voltage drop is sufficient to reveal the nonlinear part of $I-V$ characteristics.

In Table I we present values of maximal resistance $R$ at room temperature and show the corresponding parameters of the tunneling barrier derived from Brinkman fits ${ }^{15}$ commonly used for trapezoidal barriers. The calculated barrier heights $\varnothing$ vary from 0.33 to $0.78 \mathrm{eV}$ for different junctions, which all show a definite barrier asymmetry. The variation of the barrier heights could be related to locally different $\mathrm{Si}$ contents in the Si-rich spacer. Actually, as was shown previously, an increase of the nominal Si content in a spacer layer to more than $70 \%$ leads to an increase of the mean barrier height $\varnothing$ from 0.15 to $0.7 \mathrm{eV}^{4}$ The observed barrier asymmetry is most likely caused by different rates of diffusion at $\mathrm{Fe} / \mathrm{Si}$ and $\mathrm{Si} / \mathrm{Fe}$ interfaces. ${ }^{5,7,16}$ In the case of interdiffusion, the effective thickness $t_{\mathrm{eff}}$ of the tunneling barriers should become smaller than the nominal value $t$. However, for junctions $J 1$ and $J 3$ the Brinkman fits result in $t_{\text {eff }}>t$, which according to Ref. 11 is an indication of a small amount of pinholes in the spacer layer. In this case, the transport across the pinholes coexists with the dominating tunneling process and leads to a slightly decreased value of the fitted barrier height. ${ }^{11}$ Actually, for junctions $J 1$ and $J 3$ the fitted mean barrier heights turn out to be comparatively small (Table I).

A typical temperature dependence of the zero-bias resistance for junctions with $t_{\text {eff }}<t$ is presented in Fig. 5. The resistance slightly decreases with temperature and shows tunneling-type behavior. The corresponding total change of resistance does not exceed $5 \%-7 \%$. We relate the change of resistance to prevailing direct elastic tunneling, which yields a weak temperature dependence due to the broadening of
Fermi distributions. The elastic but resonant tunneling channel is much weaker than the direct one and obeys a decay length, which is twice as large as the decay length of direct tunneling. However, resonant elastic tunneling cannot definitely be excluded for our junctions with $t$ lying between 1.4 and $1.7 \mathrm{~nm}$. Different weights of the contributions from elastic direct and elastic resonant tunneling could lead to the scattering of the $R A$ values in Fig. 4. Next, we consider inelastic tunneling based on thermo-activated hopping across impurity states in the barrier. For this channel a strong decrease of resistance with temperature is expected. ${ }^{14}$ Thus, this channel is not dominant in our junctions. Finally, the third criterion for direct tunneling - the negative temperature coefficient of the resistance observed in Fig. 5-allows us to exclude a significant metallic contribution to the electron transport through pinholes for a subset of our junctions ( $J 2$, $J 4, J 5$, and J6).

\section{CONCLUSIONS}

We conclude that epitaxial, AF coupled Fe/Si/Fe junctions fulfil the necessary and sufficient criteria for direct elastic electron tunneling: The junctions show (i) a strong increase of area times resistivity product with spacer thickness, (ii) parabolic conductivity versus voltage dependencies, and (iii) small negative temperature coefficients of the resistance. Thus, we could show for a set of AF coupled Fe/Si/Fe junctions that there is no significant contribution to the conductivity caused by pinholes. The experimentally proven coexistence of both strong AF coupling and electron transport via elastic tunneling across nominally pure Si spacers is an important piece of information for a better understanding of the mechanism of strong AF interlayer exchange coupling across Si spacers.

${ }^{1}$ E. E. Fullerton, J. E. Mattson, S. R. Lee, C. H. Sowers, Y. Y. Huang, G. Felcher, and S. D. Bader, J. Magn. Magn. Mater. 117, L301 (1992).

${ }^{2}$ K. Inomata, K. Yusu, and Y. Saito, Phys. Rev. Lett. 74, 1863 (1995).

${ }^{3}$ J. J. de Vries, J. Kohlhepp, F. J. A. den Broeder, R. Coehoorn, R. Jungblut, A. Reinders, and W. J. M. de Jonge, Phys. Rev. Lett. 78, 3023 (1997).

${ }^{4}$ Y. Endo, O. Kitakami, and Y. Shimada, J. Appl. Phys. 87, 6836 (2000).

${ }^{5}$ R. R. Gareev, D. E. Bürgler, M. Buchmeier, R. Schreiber, and P. Grünberg, J. Magn. Magn. Mater. 240, 237 (2002).

${ }^{6}$ R. R. Gareev, D. E. Bürgler, M. Buchmeier, D. Olligs, R. Schreiber, and P. Grünberg, Phys. Rev. Lett. 87, 157202 (2001).

${ }^{7}$ R. R. Gareev, D. E. Bürgler, M. Buchmeier, R. Schreiber, and P. Grünberg, Appl. Phys. Lett. 81, 1264 (2002).

${ }^{8}$ R. R. Gareev, D. E. Bürgler, M. Buchmeier, R. Schreiber, and P. Grünberg, Trans. Magn. Soc. Jpn. 2, (2002).

${ }^{9}$ P. Bruno, Phys. Rev. B 52, 411 (1995).

${ }^{10}$ D. A. Rabson, B. J. Jönsson-Åkerman, A. H. Romero, R. Escudero, C. Leighton, S. Kim, and I. K. Schuller, J. Appl. Phys. 89, 2786 (2001).

${ }^{11}$ J. J. Åkerman, R. Escudero, C. Leighton, S. Kim, D. A. Rabson, R. W. Dave, J. M. Slaughter, and I. K. Schuller, J. Magn. Magn. Mater. 240, 86 (2002)

${ }^{12}$ R. J. M. van de Veerdonk, J. Novak, R. Meservey, J. S. Moodera, and W. J. M. de Jonge, Appl. Phys. Lett. 71, 2839 (1997).

${ }^{13}$ J. S. Moodera and G. Mathon, J. Magn. Magn. Mater. 200, 248 (1999).

${ }^{14}$ Y. Xu, D. Ephron, and M. R. Beasley, Phys. Rev. B 52, 2843 (1995).

${ }^{15}$ W. F. Brinkman, R. C. Dynes, and J. M. Rowell, J. Appl. Phys. 41, 1915 (1970).

${ }^{16}$ R. Kläsges, C. Carbone, W. Eberhardt, C. Pampuch, O. Rader, T. Kachel, and W. Gudat, Phys. Rev. B 56, 10801 (1997). 